\title{
Diagnostic communautaire en santé : Etude transversale dans la ville de Konobougou, Mali
}

\section{Community diagnosis in health: Cross-sectional study in the city of Konobougou, Mali}

\author{
Coulibaly $\mathrm{MB}^{1 *}$, Tembiné $\mathrm{I}^{1}$, Coulibaly $\mathrm{D}^{2}$, Diarra $\mathrm{M}^{3}$, Maïga $\mathrm{M}^{4}$, Dicko $\mathrm{F}^{5,6}$
}

\section{DOI : 10.53318/msp.v11i1.1897}

1 : Centre Santé Communautaire et Universitaire de Konobougou (Ségou-Mali)

2 : Centre de santé de référence de Bla (Ségou-Mali)

3 : Centre de santé de référence de Barouéli (Ségou-Mali)

4 : Projet Communautés Locales d'Enseignement pour les Femmes et les Filles en Santé (Bamako-Mali)

5 : Service de pédiatrie au CHU de Gabriel Touré (Bamako-Mali)

6 : Département de médecine de famille/médecine communautaire ; FMOS (Bamako-Mali)

*Auteur correspondant : Dr Mamadou Bayo Coulibaly au Centre Santé Communautaire et Universitaire (CSCom-U) de Konobougou, tel : 00223 $\begin{array}{llllllllllll}76 & 06 & 78 & 82 / & 00 & 223 & 69 & 74 & 97 & 76 & \text {; }\end{array}$ bayo_coul@yahoo.fr/bayo_coul@hotmail.com

\section{Résumé}

Introduction : Afin d'améliorer efficacement la santé communautaire, il est nécessaire d'établir une stratégie de promotion de la santé basée sur les besoins communautaires. L'objectif de ce travail était d'identifier les problèmes de santé prioritaires de la population de Konobougou pouvant servir de base à une stratégie d'amélioration des conditions de santé dans cette ville. Matériel et méthodes : II s'agissait d'une étude transversale descriptive allant d'Août à Septembre 2018 dans la ville de Konobougou qui est le chef-lieu de la commune du même nom au Mali. La taille de l'échantillon était de 50 participants. L'enquête concernait les chefs de famille, les mères de familles et les propriétaires des concessions. Résultats : Les personnes enquêtées étaient constituées de $92 \%$ d'hommes et $8 \%$ de femmes ; $88 \%$ étaient propriétaires de la maison et $12 \%$ étaient chefs de ménages. Les familles utilisaient comme eau de boisson les robinets dans $90 \%$ des cas et le puits dans $10 \%$ des cas. Seulement $8 \%$ des familles avaient une poubelle mais on notait une absence de couvercle dans $75 \%$ des cas sur ces poubelles. Le paludisme était la maladie la plus rencontrée avec $76 \%$ suivi des infections respiratoires aigües avec $12 \%$ des cas dans les familles selon les enquêtés. Conclusion : Les problèmes de santé dans cette ville étaient liés aux mauvaises conditions d'hygiène et d'assainissement qui seraient à l'origine de certaines maladies telles que : le paludisme, les infections respiratoires aigües et la diarrhée. II paraît donc nécessaire d'orienter notre action sur la promotion de l'hygiène et l'assainissement.

Mots clés : Diagnostic communautaire, santé, Konobougou, Mali

\footnotetext{
Abstract

Introduction: In order to effectively improve community health, it is necessary to establish a health promotion strategy based on their needs. The aim of this work was to
}

identify the priority health problems of the population of Konobougou which could serve as a basis for a strategy to improve health conditions in this city. Material and methods: This was a cross-sectional and descriptive study from August to September 2018 in the city of Konobougou, which is the capital of the commune of the same name in Mali. The sample was at 50 covering heads of households, mothers and owners of concessions. Results: The people surveyed consisted of $92 \%$ men and $8 \%$ women; $88 \%$ owned the house and $12 \%$ are heads of household. The families used the taps $90 \%$ and the well $10 \%$ of the time for drinking water. Only $8 \%$ of families had a trash can but there was an absence of a cover $75 \%$ of the cases on these trash cans. Malaria was the disease most encountered at $76 \%$ followed by acute respiratory infections in $12 \%$ of cases in families according to the people surveyed. Conclusion: The health problems in this city were based on hygiene and sanitation which would be at the origin of diseases such as: malaria, acute respiratory infections and diarrhea. It therefore seems necessary to orient our action on hygiene and sanitation promotion.

Keywords: Community diagnosis, health, Konobougou, Mali

\section{Introduction}

Ainsi, le diagnostic communautaire par définition permet de mieux connaître la nature des dysfonctionnements au sein d'un groupe en s'intéressant aux origines de ceux-ci, il permet également l'évaluation de la dynamique locale sur laquelle le travail des professionnels pourra s'appuyer (1).

Au tournant des années 1970, un peu partout dans le monde, l'expression "santé communautaire » était très présente dans l'univers de la santé publique et plus largement dans celui du socio-sanitaire (2). Elle est inspirée de sources principalement latino-américaines et américaines (2). Pendant ces périodes, une fracture s'est faite entre les pays. Certains pays proposaient d'une part, une stratégie sanitaire basée sur les approches collectives de la santé mettant l'accent sur la prévention et accompagnées par des soins médicaux " de base " accessibles à tous. Alors que d'autres pays ont massivement financé une médecine hautement technologique, laissant peu de places à la prévention et s'investissant finalement peu à comprendre et résoudre les mécanismes d'un environnement générateur de maladies (3). Selon l'Organisation Mondiale de la Santé (OMS), la santé communautaire est le processus par lequel les membres d'une collectivité, géographique ou sociale, conscients de leur appartenance à un même groupe, réfléchissent en commun sur les problèmes de 
leur santé, expriment leurs besoins prioritaires et participent activement à la mise en place, au déroulement et à l'évaluation des activités les plus aptes à répondre à ces priorités $(2,3,4)$.

L'évaluation des besoins de santé de la communauté représente le premier pas de la prise en charge communautaire. Comme dans la médecine individuelle classique, il faut comprendre quel est le problème de la communauté (5). Le diagnostic permet d'une part de souligner l'importance de l'environnement de santé, d'autre part d'éclairer les solutions pour la promotion de la santé de ces communautés. Afin d'améliorer efficacement la santé de la communauté, il est nécessaire d'établir une stratégie de promotion de la santé à partir de leurs besoins $(6,7)$. La création d'un partenariat entre les chercheurs, les acteurs, et la population pourrait être une alternative efficace pour la résolution de problème de santé (8).

La plupart des diagnostics communautaires sont mis en œuvre dans des milieux urbains. Là où, comme l'indique le chercheur Michel Joubert, l'approfondissement de la crise économique et sociale à partir des années $80 \mathrm{a}$ rejailli très directement sur les populations les plus défavorisées (1). Une telle étude est très rarement menée au Mali et n'a jamais été réalisée à Konobougou. C'est ainsi que nous avons mené cette étude dans une commune rurale précisément dans la ville de Konobougou qui est son chef-lieu de la commune. L'objectif de ce travail était d'identifier les problèmes de santé prioritaires de cette population pouvant servir de base à une stratégie d'amélioration des conditions de santé dans cette ville.

\section{Matériel et méthodes}

II s'agissait d'une étude transversale descriptive allant d'Août à Septembre 2018. L'étude s'est déroulée dans la ville de Konobougou qui est le chef-lieu de la commune du même nom au Mali. La population de Konobougou était de 9642 habitants en 2018.

L'étude concernait les chefs de famille, les mères de famille et les propriétaires des concessions. La taille de l'échantillon était de 50. Nous avons pris 13 familles par quartier pour les deux grands quartiers et 12 familles par quartiers pour les deux petits. Nous avons sélectionné la troisième famille dans les rues prises au hasard.

Les données ont été collectées dans les familles par quatre équipes de deux personnes dans les quatre quartiers de Konobougou. Un questionnaire préétabli a été renseigné par l'interview avec les enquêtés plus l'observation faite par les enquêteurs. Les données ont ensuite été traitées et analysées par Epi info version 3.5.3. Les variables étudiées étaient : les renseignements généraux des familles, l'approvisionnement en eau potable, l'hygiène assainissement (corporelle par le lavage des mains à différent moment et environnemental), l'évacuation des ordures ménagère et des eaux usées, la lutte contre les insectes et les rongeurs, la nutrition des enfants de moins de cinq ans, les maladies couramment rencontrées dans les familles, l'état vaccinal des femmes enceintes et des enfants de 0 à 5 ans. Les différents moments de lavage des mains étaient : avant de manger, de donner à manger à l'enfant, après avoir quitté la toilette ou après avoir fait la toilette de l'enfant et avant de cuisiner. Ici un bon état de la maison était des maisons avec des fenêtres, des portes plus les toitures et sans fissures.

Les résultats obtenus ont été discutés au cours d'une rencontre avec les autorités administratives et communales, les leaders coutumiers et la communauté afin de les conscientiser et les priorités ont été définies. Des recommandations ont été faites pour faire face aux priorités (le paludisme, les infections respiratoires et les accidents de la voie publique).

Un consentement individuel verbal de chaque participant à l'étude a été obtenu. L'étude n'avait aucun risque additionnel chez les personnes. Seul un numéro d'identification a été utilisé. Les données ont été gardées de façon confidentielle.

\section{Résultats}

Cette étude portait sur 50 concessions dans la ville de Konobougou, les personnes enquêtées étaient constituées de $92 \%$ d'hommes et $8 \%$ de femmes.

Sur les familles enquêtées, $88 \%$ étaient propriétaires de maison et $12 \%$ étaient des chefs de ménages. Les enquêtés ont déclaré que 3 personnes dormaient par chambre en moyenne. Les maisons étaient en bon état dans $84 \%$ des cas (tableau I). Dans toutes les maisons soit $100 \%$, il existait des portes et des fenêtres. II y avait la présence de parc d'animaux dans les concessions dans $64 \%$ des cas et de culture dans $12 \%$ des cas (tableau I). Les $90 \%$ des familles utilisaient l'eau de robinets pour leurs boissons à travers la SOMAGEP. L'eau de puits était utilisée dans $10 \%$ des cas pour la boisson et $100 \%$ des cas pour d'autres fins. Les puits qui avaient une margelle représentaient $80 \%$, aucun n'avait de revêtement, la présence de couvercle et de suspensoir était noté dans $70 \%$ des cas chacun et $76 \%$ des puits bénéficiaient d'un traitement. Au total, $94 \%$ des familles conservaient leurs eaux dans des jarres qui possédaient toutes des fermetures. Les eaux stockées par les familles prises dans les puits pour usage de boisson étaient traitées avec l'eau de javel dans $80 \%$ des cas. Le renouvèlement de ces eaux stockées était réalisé deux à trois fois par semaine dans $58 \%$ des cas.

Seulement $8 \%$ des familles avaient une poubelle mais on notait une absence de couvercle sur $75 \%$ de ces poubelles. La totalité des familles avaient un dépôt d'ordure, mais aucune n'avait un circuit de collète, ou d'évacuation finale des ordures. Les $60 \%$ familles déversaient leurs eaux usées dans la rue et $28 \%$ la déversaient dans la cour. Le compostage était utilisé par $92 \%$ des familles comme méthode de traitement des ordures. Ces ordures étaient enlevées de façon semestrielle dans $90 \%$ des cas et amenées au champ pour servir de fumier. La distance puits-latrine était respectée dans $94 \%$ des familles. 
Le lavage des mains dans les concessions à différents moments était à $68 \%$ avant et après avoir mangé, à $70 \%$ après avoir quitté la toilette des enfants et $72 \%$ après la toilette.

Les $98 \%$ familles enquêtées utilisaient les moustiquaires imprégnées d'insecticide pour lutter contre les insectes notamment les moustiques et les $84 \%$ des rodonticides. L'allaitement maternel exclusif avant six mois était utilisé dans $80 \%$ des familles et $52 \%$ des mères commençaient la supplémentation alimentaire à six mois (tableau II). Les enfants étaient ablactés à l'âge de deux ans ou plus dans $96 \%$ des cas (tableau II). Le mode de d'ablactation était brusque dans $86 \%$ des cas. En cas de grossesse rapprochée, $82 \%$ des mères faisaient l'alimentation mixte c'est-à-dire le sevrage.

Face à des maladies, $86 \%$ des familles faisaient recours à une structure de santé (figure I).

La vaccination des enfants était à jour dans $98 \%$ des cas et $96 \%$ des cas pour les femmes enceintes. Le paludisme était la maladie plus rencontrée avec $76 \%$, suivi des infections respiratoires aigües avec $12 \%$ selon les personnes enquêtées (figure II).

\section{Discussion}

Limites : les maisons récemment construites en périphérie non pas été prises en compte.

Force : l'adhésion de la communauté à cette étude.

Sur les familles enquêtées, la plus part était propriétaires de maison. Cela s'expliquerait par le fait qu'en milieu rural le système de loyer n'est très fréquent, et s'il est pratiqué le propriétaire de maison vie en général avec les locataires.

Les enquêtés ont déclaré que 3 personnes dormaient par chambre en moyenne. Tandis qu'Edward D M et al. trouvent que la promiscuité est propice aux troubles du sommeil, au comportement turbulent des enfants (9). Cette idée avait aussi été soutenue par Faya J (10).

Les maisons étaient en bon état dans la plus part des cas. Dans toutes les maisons il existait des portes et des fenêtres. Ces résultats dénoteraient des signes qui seraient protecteurs de la communauté contre certaines maladies en empêchant les rongeures et les insectes de pénétrer dans les maisons sans oublier l'air frais (le vent froid) pendant la période froide.

II y avait la présence de parc d'animaux dans les concessions dans près de deux tiers et de culture dans plus d'un dixième de cas. Ces données favoriseraient la prolifération des mouches, des moustiques et d'autres vecteurs des maladies diarrhéiques et du trachome.

Les familles utilisaient l'eau de robinets pour leurs boissons à travers la SOMAGEP dans la majorité des cas et l'eau de puits dans la totalité des cas pour d'autres fins. Les puits qui avaient une margelle représentaient plus des trois quarts des cas, on avait noté une absence de revêtement dans tous des cas, la présence de couvercle et de suspensoir était noté dans plus de deux tiers des cas chacun et environ les trois quarts des puits bénéficiaient d'un traitement. Les familles conservaient leur eau dans la jarre dans la quasi-totalité des cas et la totalité possédait une fermeture. Les eaux stockées prises dans les puits pour usage de boisson étaient traitées avec l'eau de javel dans la majorité des cas. Le renouvèlement de ces eaux stockées était réalisé deux à trois fois par semaine dans à peu près plus de la moitié des cas. La consommation de l'eau potable associée aux mesures qui la protègent permettraient à la population d'éviter des maladies comme le choléra, la dracunculose et d'autres diarrhéiques. Alors que Rouamba $\mathrm{J}$ et al. trouvent que malgré les infrastructures mises en place après le lotissement du quartier au Burkina Faso en 2002, le problème d'accès à l'eau potable restait entier, surtout pour les populations de la zone non aménagée dont $73 \%$ étaient à plus de 500 mètres des bornes fontaines (11). Tandis que Bazié J B avait dit qu'hormis l'Afrique du Nord et une partie de l'Afrique du Sud, la plupart des pays du continent sont confrontés au défi d'une gestion performante des services d'eau et d'assainissement en zone urbaine comme en zone rurale (12).

Seulement moins d'une dixième des familles avaient une poubelle, mais on notait une absence de couvercle dans les trois quarts des cas sur ces poubelles. La totalité des familles avaient un dépôt d'ordure, mais pas de circuit de collecte et ni l'évacuation finale des ordures dans la totalité des cas. Les familles déversaient leur eau usée dans la rue dans plus de la moitié des cas et environ un quart des familles la déversaient dans la cour. Le compostage était utilisé par les familles dans presque totalité des cas comme méthode de traitement des ordures dans la ville. Ces ordures étaient enlevées de façon semestrielle dans la majorité des cas et amenées au champ pour servir de fumier. La distance puits-latrine était respectée par la quasi-totalité des familles. Cela démontrait que l'environnement où vit la communauté n'était aussi bien salubre. L'insalubrité serait en faveur du développement des mouches et des cafards vecteurs des maladies telles que la diarrhée, la fièvre typhoïde et les maladies respiratoires. Cependant, Carlemagne et al. trouvent que l'environnement insalubre pouvait être à l'origine de pathologies spécifiques (morsures de rats, dermatoses, fragilité respiratoire liée à la pollution atmosphérique) (13). Tandis que l'accès à l'assainissement était un enjeu majeur de santé publique, en particulier dans les pays en voie de développement selon Morin H (14).

Le lavage des mains dans les concessions à différents moments était plus de la moitié avant et après avoir mangé, près de trois quart après avoir fait la toilette des enfants et après avoir quitté la toilette. Le résultat souhaité était $80 \%$ ou plus de lavage à différents moments dans les ménages. La maîtrise de ce geste permettrait d'empêcher la contamination de soi-même et interhumaine contre les maladies liées à l'insalubrité.

Les familles enquêtées utilisaient les moustiquaires imprégnées d'insecticide pour lutter contre les insectes notamment les moustiques dans la quasi-totalité des cas les rodonticides contre les rongeurs. Ces moyens de lutte contre les rongeurs et insectes sont simples et efficaces 
s'ils sont correctement utilisés par la communauté. Cependant une attention doit être accordée aux insecticides pour ne pas entrainer une intoxication. Alors que Cherin $P$ et al considéraient que ces produits phytosanitaires possèdent tous une toxicité, d'intensité variable, pour l'homme s'il y a une mauvaise utilisation, un usage accidentel ou une intoxication volontaire souvent gravissime (15).

L'allaitement maternel exclusif avant six mois était le plus utilisé dans la majorité des cas et un peu plus de la moitié des mères commençaient la supplémentation alimentaire à six mois. Les enfants étaient ablactés à l'âge de deux ans ou plus dans la quasi-totalité des cas. Le mode de l'ablactation était brusque dans la majorité des cas. En cas de grossesse rapprochée, la majorité des mères faisaient l'alimentation mixte. L'allaitement maternel exclusif avant six mois et la supplémentation en cas de grossesse rapprochée plus la période de sevrage étaient en phasage avec les normes. Mais des difficultés ont été constatées dans la supplémentation à partir de six mois et le mode de sevrage qui seraient responsables de malnutrition aigüe car à six mois le lait maternel seul n'apporte pas tous ceux dont l'organisme de l'enfant a besoin, le refus de s'alimenter pourrait s'ajouter quand l'enfant n'est pas habitué à d'autres aliments que le lait de sa mère. Alors que chaque année, la malnutrition est impliquée dans près de $40 \%$ des 11 millions de décès d'enfants de moins de cinq ans dans les pays en développement, et on estime que 1,5 millions de ces décès pourraient être évités par la pratique de l'allaitement maternel immédiat et exclusif pendant la période néonatale selon Traoré $\mathrm{M}$ et al. (16).

Face à des maladies, les trois quarts des familles faisaient recours à une structure de santé. La vaccination des enfants et des femmes enceintes étaient à jour dans la presque totalité des cas. Le paludisme était la maladie la plus rencontrée dans plus des trois quarts des cas suivi des infections respiratoires aigües dans plus d'un dixième des cas. Le recours aux structures de santé permettrait d'éviter des complications et des maladies évitables par le programme élargi de vaccination. Ces comportements doivent être soutenus et continuels. Tandis que dans les indicateurs démographiques et de santé de la Guinée plusieurs maladies transmissibles étaient ciblées comme prioritaires dont le groupe $\mathrm{VIH}$ (virus de l'immunodéficience humaine) /sida (syndrome d'immunodéficience acquise), tuberculose, paludisme et plus récemment Ebola selon Vieira $G$ et al. (17).

Dans le registre de consultation du centre de santé communautaire et universitaire, le paludisme occupait la première place suivi des IRA et les AVP soit respectivement $62,45 \%, 12,87 \%$ et $7,87 \%$. Alors que dans l'étude de Vieira $\mathrm{G}$ et al, les informations recueillies dans les établissements hospitaliers publics en Guinée en 2012 ont indiquée comme principales causes de mortalité : le paludisme, les infections respiratoires, les maladies cardiovasculaires, les tumeurs et les complications liées à l'accouchement chez les adultes (17).

\section{Conclusion}

Les problèmes de santé dans cette ville étaient basés sur les mauvaises conditions d'hygiène et d'assainissement qui seraient à l'origine de certaines maladies telles que : le paludisme, les infections respiratoires aigües, la diarrhée et autres. II paraît donc nécessaire d'orienter notre action pour la promotion d'hygiène et d'assainissement.

\section{Conflit d'intérêt : aucun}

\section{Références}

1. Bantuelle M, Morel J, Dargent D. Le diagnostic communautaire. L'asbl "Santé, Communauté, Participation", 34p.

http://labos.ulg.ac.be/apes/wpcontent/uploads/sites/4/2014/05/diagncommunautaire.pdf

2. Jordan D, O'Neill M, Dupéré S, Stirling J. Quarante ans après, où en est la santé communautaire ? Santé Publique 2012 ; 24 : 165178.

3. Motamed S. Qu'est-ce que la santé communautaire ? Un exemple d'une approche participative et multisectorielle dans une commune du Canton de Genève, en Suisse. Information psychiatrique $2015 ; 91: 563-567$.

4. Michel B. La santé communautaire aujourd'hui. Revue du soignant en santé publique 2007 ; 4 : 1215.

5. Jaunin-Stalder N, Pécoud A. Approche communautaire en médecine de premier recours. Primary Care $2007 ; 7: 357-360$.

6. Vieira G, Courtois R, Rusch E. Approche d'autonomisation d'une communauté africaine dans le diagnostic de soins de santé de deux pays: la Guinée Conakry et le Congo Brazzaville. Pan Afr Med J 2017 ; 28 : 276.

7. André-Anne Parent, Michel O'Neill, Bernard Roy, Paule Simard. Entre santé publique et organisation communautaire : points de convergence et de divergence autour du développement des communautés au Québec. Revue de l'Université de Moncton $2012 ; 43$ : 67-90.

8. Sebbani M, Adarmouch L, Amine M, Cherkaoui M. Mobilisation communautaire pour l'amélioration de la surveillance de la grossesse en milieu rural au Maroc. Pan African Medical Journal. 2020;35:73.

9. Edward D M, Watt R G. Dietand hygiene in the lives of Gypsy Travellers in Hertfordshire. Community Dent Health 1997 ; 14(1) :41-6.

10. Faya J. Diagnostic de sante communautaire de la population des «Gens du Voyage» du Rhône. 2001, $17 p$. https://www.angvc.fr/wpcontent/uploads/2016/06/ARTAG-MDM-2001.pdf

11. Rouamba J, Nikiema E, Rouamba S, De Charles Ouedraogo F. Accès à l'eau potable et risques 
sanitaires en zone périphérique de Ouagadougou, Burkina Faso. Revue d'Épidémiologie et de Santé Publique 2016 ; 64 : S211.

12. Bazié J B. Accès à l'eau : l'Afrique entre abondance et pénurie. Après demain 2014 ; 3-4 : 28-29.

13. Charlemagne J, Khan K, Querouil O, Guiraud J C, Lussault M, Bouillon F et al. Tsiganes et santé, de nouveaux risques? Revue d'Etudes Tsiganes $2000 ; 14: 184$

14. Morin H, Arfi C, Detournay B, Gilbert O, Joseph $\mathbf{X}$, Chagraoui M. Étude de l'impact sanitaire du programme d'amélioration de l'accès à l'eau potable et de l'assainissement à Tanger au Maroc. Revue d'épidémiologie et de santé publique 2010 ; 58 : S60.

15. Cherin $P$, Voronska $E$, Fraoucene $N$, de Jaeger C. Toxicité aiguë des pesticides chez l'homme. Médecine et longévité $2012 ; 4(2), 68-74$.

16. Traore M, Sangho H, Diagne M C, Faye A, Sidibé $\mathrm{A}$, Kone $\mathrm{K}$ et al. Facteurs associés à l'allaitement maternel exclusif chez les mères d'enfants de 24 mois à Bamako 2014 ; Santé publique 2014 ; 26 : 259-265.

17. Vieira G, Courtois R, Rusch E. Approche d'autonomisation d'une communauté africaine dans le diagnostic de soins de santé de deux pays: la Guinée Conakry et le Congo Brazzaville. Pan Afr Med J 2017; 28: 276.

Tableaux et figures

Tableau I : L'état des maisons plus l'existence de culture et de parc dans les concessions à Konobougou

Effectif Pourcentage

\begin{tabular}{llcc}
\hline \multicolumn{2}{l}{ Etat des maisons } & & \\
& Bon & 42 & 84 \\
& Mauvais & 8 & 16 \\
$\begin{array}{l}\text { Présence de } \\
\text { portes et de } \\
\text { fenêtres }\end{array}$ & & & \\
& Oui & 50 & 100 \\
Parcage dans & Non & 0 & 0 \\
$\begin{array}{l}\text { les } \\
\text { concessions }\end{array}$ & & & \\
& Présent & 32 & 64 \\
Culture dans & Absent & 18 & 36 \\
les & & & \\
concessions & & & \\
& Présente & 6 & 12 \\
& Absente & 44 & 88 \\
\hline
\end{tabular}

Tableau II : L'alimentation des enfants et l'âge de sevrage des enfants à Konobougou

\begin{tabular}{lcc}
\hline & Nombre & Pourcentage \\
\hline Allaitement des nourrissons & avant 6 mois \\
Exclusif & 40 & 80 \\
Mixte & 7 & 14 \\
Artificiel & 3 & 6 \\
Supplémentation alimentaire à six mois \\
A six mois & 26 & 52 \\
Après six mois & 24 & 48 \\
Age de sevrage des enfants & \\
Deux ou plus & 48 & 96 \\
Un an & 2 & 4 \\
\hline
\end{tabular}

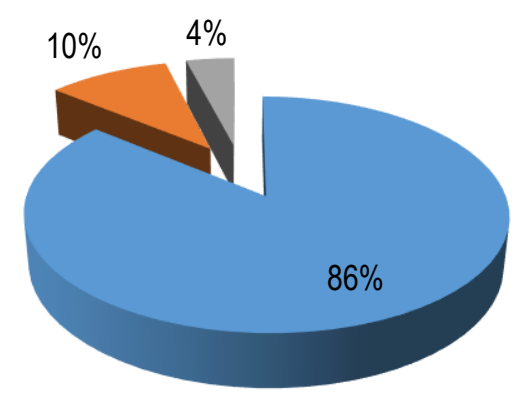

Recours à une structure de santé

- Traitement traditionnel

Automédication

Figure 1 : Le premier recours des mères face aux maladies à Konobougou 


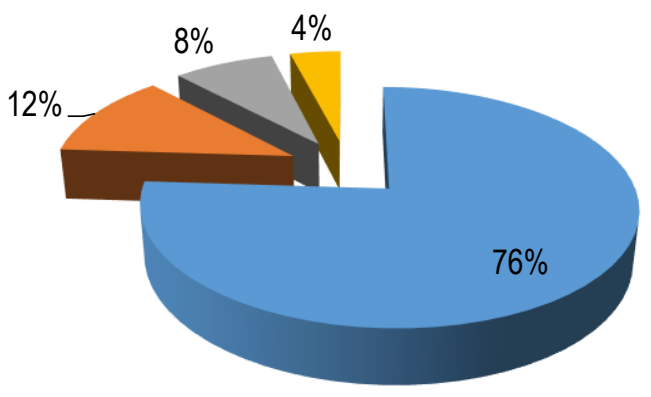

- Paludisme

- Infection respiratoire aigue

Diarrhée

Malnutrition aigue

Figure 2 : Les maladies couramment rencontrées dans les familles à Konobougou 